\title{
Review of: "Immunophenotype in Acute Exacerbation of Chronic Obstructive Pulmonary Disease: A Cross- Sectional Study"
}

\author{
Teresa Torres Moral
}

Potential competing interests: The author(s) declared that no potential competing interests exist.

Congratulations to all the authors for this interesting study. The study analyses the immunophenotype of patients with acute exacerbation of Chronic obstructive pulmonary disease (AECOPD), and the results are compared with healthy controls and with stable Chronic obstructive pulmonary disease (SCOPD). Authors measured, in peripheral blood, CD4+ T lymphocyte subtypes (Th1, Th2, Th17 and Treg) through flow cytometry and the levels of serum cytokines through a plasma cytokine assay. A questionnaire and a pulmonary function test were also conducted to evaluate the severity of the illness. The results were that AECOPD patients show abnormal activation of Th1, Th17 and Treg cells and that there is a correlation between the proportion of Th17 cells and the severity of COPD.

A useful flowchart facilitates the understanding of the pipeline of the study

From my point of view, this is a compelling article but there are some small concerns that, in my opinion, could be useful to clarify:

1. About the experimental process of the flow cytometry, it could be better to mention the source of the protocol that you follow (even if it is the manufacturer's indications)

2. Taking into account that the relationship of TH17 with the severity of COPD has not been extensively studied and that other studies looking for this relationship have not found it

(e.g. https://pubmed.ncbi.nlm.nih.gov/28934595/ does not find a relation between Th17 proportion and GOLD score ), I suggest you to be cautious when expressing possible use of Th17 proportion as an index for evaluation of COPD severity. Also, it could be useful to mention those studies in the 'Discussion' section trying to grasp the differences in results.

3. English is perfectly understandable, but there are some typo mistakes (ex. Page 3-Material and Methods-Subject: “(17]. patients and controls were recruited according to.."). A review of the text could improve the correctness of the article. 